\title{
Changes in Body Composition and Cardiometabolic Health After Detraining in Older Men with Osteosarcopenia: 6-Month Follow-Up of the Randomized Controlled Franconian Osteopenia and Sarcopenia Trial (FrOST) Study
}

This article was published in the following Dove Press journal: Clinical Interventions in Aging

\author{
Wolfgang Kemmler $\mathbb{D}^{1}$ \\ Daniel Schoene (D) \\ Matthias $\mathrm{Kohl}$ (D) $^{2}$ \\ Simon von Stengel ${ }^{1}$ \\ 'Institute of Medical Physics, Friedrich- \\ Alexander University of Erlangen- \\ Nürnberg, Erlangen, 91053, Germany; \\ ${ }^{2}$ Faculty Medical and Life Sciences, \\ University of Furtwangen, Villingen- \\ Schwenningen, 78054, Germany
}

Purpose: Temporary cessation of exercise but maintenance of habitual physical activity might be a frequent situation in older people's lives. Particularly the COVID-19 induced lockdown of exercise training facilities with individual outdoor activities still being allowed might be a blueprint for this potentially harmful scenario. Thus, the aim of the present study was to determine the effects of 6 months of detraining after 18 months of high-intensity resistance exercise (HIT-RT) on body composition and cardiometabolic outcomes in predominately obese older men with osteosarcopenia.

Materials and Methods: Community-dwelling predominately obese men $72-91$ years old with low muscle and bone mass $(n=43)$ were randomly assigned to an 18-month HIT-RT (EG: $n=21)$ or a non-training control group $(\mathrm{CG}, \mathrm{n}=22)$. After the intervention, participants of the EG discontinued HIT-RT for 6 months, but increased their habitual physical activity. Study outcomes were group differences in detraining changes ("effects") for lean body mass (LBM), total and abdominal body fat rate (determined by dual-energy x-ray absorptiometry) and the Metabolic Syndrome Z-Score (MetSZ). We applied an intention-to-treat analysis with multiple imputation to analyze the data.

Results: After the 18-month HIT-RT, we observed significant positive training effects for LBM, total and abdominal body fat rate and the MetSZ (all $\mathrm{p}<0.001$ ). Abrupt cessation of HIT-RT for 6 months resulted in significantly higher unfavorable changes in the HIT-RT compared with the CG for $\operatorname{LBM}(\mathrm{p}=0.001)$, total body fat $(\mathrm{p}=0.003)$ and the MetSZ $(\mathrm{p}=0.003)$, apart from abdominal body fat $(\mathrm{p}=0.059)$. However, significant overall effects were still present after 24 months for LBM and body fat indices but not for the MetSZ.

Conclusion: The present study clearly indicates the unfavorable effects of 6 months of detraining after HIT-RT. Correspondingly, exercise protocols particularly for older people should focus on continuous exercise with short regeneration periods rather than on intermitted protocols with pronounced training breaks.

Keywords: resistance exercise, detraining, lean body mass, body fat, metabolic syndrome, older men

\section{Introduction}

Temporary breaks of exercise routines e.g. due to the intermitted character of an exercise program, lack of time, or simple reluctance might be a frequent situation in older adults' lives. ${ }^{1,2}$ Although exercise routines were not stopped that abruptly, the present COVID-19
Correspondence: Wolfgang Kemmler Institute of Medical Physics, FriedrichAlexander University of ErlangenNürnberg, Henkestrasse 91, Erlangen, 91052, Germany

Tel +49 9131 8523999

Fax +49 9131 8522824

Email wolfgang.kemmler@imp.unierlangen.de 
induced lockdown in winter/spring 2020 in Bavaria might serve as a blueprint for the effects of a sudden cessation of supervised group exercise training with otherwise maintained or even enhanced physical (outdoor) activity. Reviewing the literature, the vast majority of studies that focus on detraining effects in older adults (e.g. ${ }^{3-16}$ ) reported severe negative effects a mere 8 weeks after cessation of the intervention. ${ }^{3}$ However, most of the studies focus on the detraining effect on musculoskeletal outcomes after shortterm interventions (ie 12-16 weeks), while the effects on body composition and cardiometabolic parameters after resistance training (RT) periods that exceed 6 months were rarely evaluated. This refers particularly to older obese people with low muscle and bone mass, ${ }^{17,18}$ who can be considered as a high-risk group. In order to close this gap, the aim of this study was to determine the effect of 6 months of detraining after an 18-month HIT-RT intervention on body composition and cardiometabolic risk in older, predominately obese, osteosarcopenic men.

Our primary hypothesis was that changes in the HITRT vs control group during the detraining period (ie detraining effects) were significantly more pronounced in the HIT-RT group for (1.1) lean body mass (LBM, primary study outcome), (1.2) total body fat, (1.3) abdominal body fat and (1.4) metabolic syndrome Z-Score in older, community-dwelling (cdw) men with osteosarcopenia and increased body fat.

Our secondary hypothesis was that the significant training effects on (2.1) LBM, (2.2) total body fat (2.3.), abdominal body fat and (2.4) the Metabolic Syndrome Z-Score observed after 18 months of intervention will be reduced to nonsignificant levels after 6 months of detraining.

\section{Materials and Methods}

The aim of the randomized controlled FrOST (Franconian Osteopenia and Sarcopenia Trial), was to determine the effects of an 18-month HIT-RT on healthand fitness-related outcomes in cdw 73-91-year-old men with osteosarcopenia. The present detraining project (FrOST-FU) aimed to determine the effect of 6 months of detraining on outcomes addressed by FrOST. A recent publication thereby focus on detraining effects on musculoskeletal parameters, ${ }^{19}$ while the present work aimed to address cardiometabolic outcomes in the FrOST cohort. The project was initiated and conducted by the Institute of Medical Physics (IMP), University of Erlangen-Nürnberg (FAU), Germany. FroST and FrOSTFU were approved by the FAU ethics committee (4464b, $4464 \mathrm{~b}$ amendment) and the federal bureau of radiation protection (BfS, number Z 5 - 2246212 - 2017-002).
The project fully complies with the Helsinki Declaration. ${ }^{20}$ All the study participants gave their written informed consent after receiving detailed information. The studies were registered under ClinicalTrials. gov NCT03453463 (FrOST) and NCT04444661 (FrOST-FU). The exercise intervention of FrOST was conducted between June 2018 and December 2019; the observational period of FrOST-FU ranges from the end of December 2019 to mid-June 2020 (6-month followup).

\section{Participants}

The recruitment process of FrOST started in February 2018 and has been described in detail in previous articles. ${ }^{18,21,22}$ Briefly, cdw men, 72 years and older with (a) morphometric sarcopenia (skeletal muscle mass index lower than -2 SD T-Score ${ }^{23,24}$ ) and (b) osteopenia or osteoporosis (bone mineral density at LS or proximal femur lower than -1 SD T-Score ${ }^{25}$ ) were included in the study. Men (a) with diseases and/or (b) taking pharmaceutical agents (eg bisphosphonates, alfacalcidol, glucocorticoids) that either affected muscle/ bone metabolism or hinder intense resistance exercise, (c) with experience in resistance-type exercise ( $\geq 60 \mathrm{~min} /$ week previous 2 years) and (d) with alcohol abuse ( $>60$ $\mathrm{g} / \mathrm{d}$ ethanol consumption) were excluded. Of importance, the final decision about eligibility was always taken by the study physician. After application of the criteria listed above and detailed study information, the finally 43 eligible men willing to participate in FrOST were randomly allocated to an HIT-RT $(\mathrm{n}=21)$ or control $(\mathrm{CG}$, $\mathrm{n}=22$ ) group. While the $\mathrm{CG}$ was asked to maintain their physical activity/exercise (CG) habits, the HIT-RT conducted the resistance exercise training described below for 18 months (Figure 1).

In December 2019, the 18-month study intervention period ended. After 18-month FU-testing, we made an appointment with the participants to restart exercise after a short training break in February 2020. However, due to logistic and budget problems, we failed to start in February and the COVID-19 induced lockdown of all exercise facilities in March 2020 prevented all further group and/or indoor exercise programs for the next 14 weeks (up to June 2020). After the termination of the lock down, we invited participants to the 6-month detraining-follow-up (June 2020). Thirty-seven participants accepted our invitation and were assessed for 6-month detraining-FU (Figure 1). 


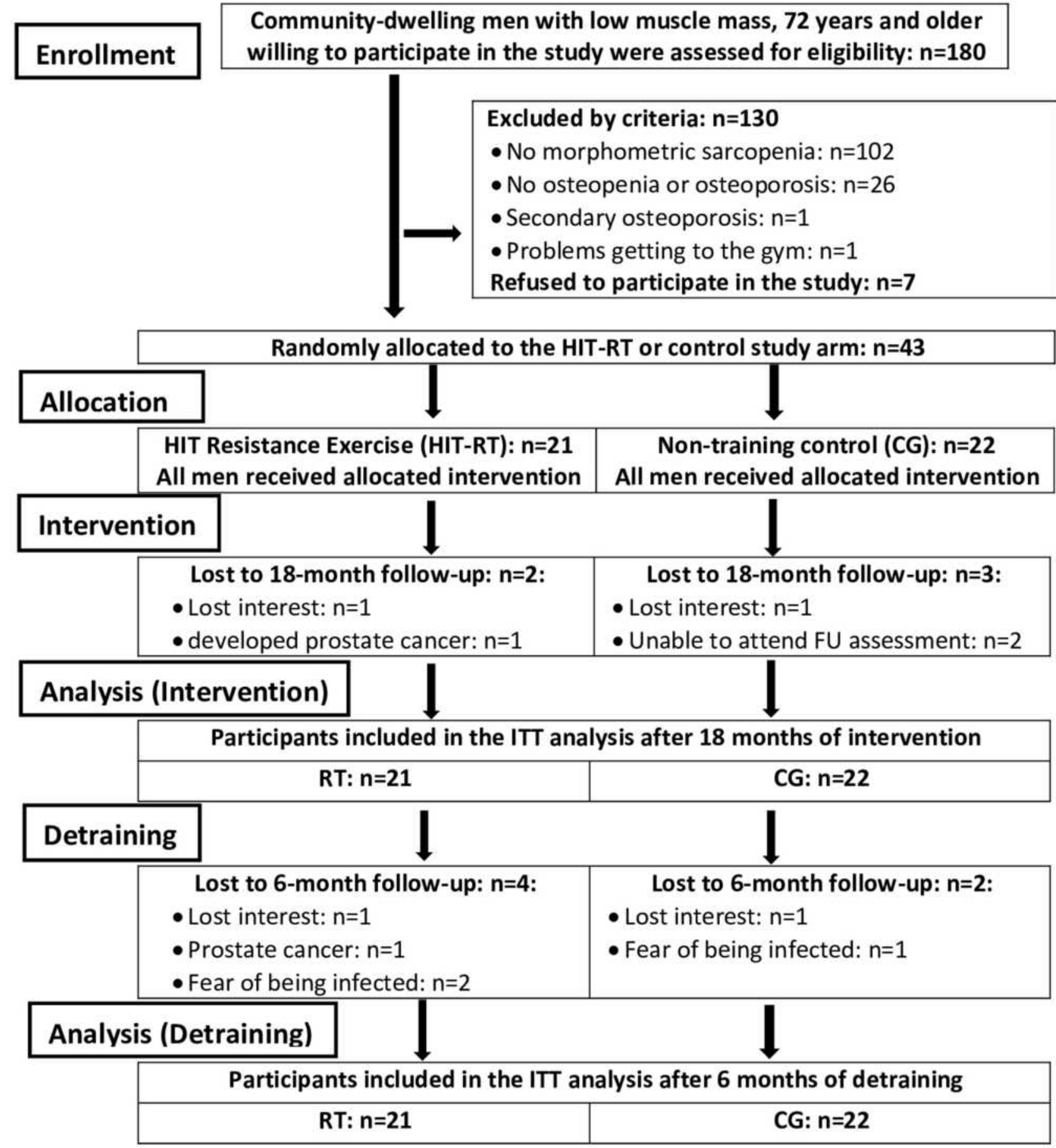

Figure I Adapted participant flow through the study.

Randomization Procedures and Blinding The randomization and blinding procedures have been described in detail in previous publications. ${ }^{18,21,22}$ Briefly, participants allocated themselves to the HIT-RT or control group by drawing lots. Of importance, neither the participants nor the researcher knew the allocation beforehand (allocation concealment). Blinding only refers to test assistants and outcome assessors who were kept unaware of the participants' group status (EG or CG).

\section{Study Procedure}

During the intervention period, all participants were provided with protein, ${ }^{26}$ Cholecalciferol and Calcium $^{27}$ as per recent recommendations. Apart from the exercise 
intervention of the HIT-RT, participants were asked to maintain their dietary habits and lifestyle including physical activity and exercise routines. Protein and calcium supplementation stopped at the end of the intervention, but all participants were provided with cholecalciferol up to the end of the detraining period.

\section{Interventions}

\section{Supplementation}

As per recent recommendations ${ }^{26}$ we provided whey protein supplementation (WPS; Active PRO80, inkospor, Roth, Germany) to generate a daily protein intake of 1.5-1.6 in the EG and $1.2-1.3 \mathrm{~g} / \mathrm{kg} / \mathrm{d}$ in the CG during the intervention period. Based on the dietary calcium questionnaire (Rheumaliga, Switzerland), we intended a calcium intake of $\approx 1000 \mathrm{mg} / \mathrm{d} .^{27}$ Participants with dietary calcium intake below $950 \mathrm{mg} / \mathrm{d}$ were provided with calcium capsules (Sankt Bernhard, Bad Dietzenbach, Germany). Based on their 25 OH Vitamin-D 3 (25-OHD) levels (ECLIA; Roche Diagnostics, Penzberg, Germany) participants were provided with cholecalciferol (MYVITAMINS, Manchester, UK). Participants with 25 OHD levels $<30 \mathrm{ng} / \mathrm{mL}$ were provided with 10,000 IE/ week and participants with OHD-level of $30-40 \mathrm{ng} / \mathrm{mL}$ were supplemented with 5000 IE/week. We verified adherence to the prescribed supplementation of protein, Vit-D and calcium by (a) checking our distribution logs; (b) biweekly phone calls and (c) personal interviews conducted at FU assessments.

\section{Resistance Exercise}

The HIT-RT intervention has been described in detail in previous publications ${ }^{18,22}$ thus only a brief overview will be provided here. In summary, we applied a progressive, periodized single set, dynamic resistance exercise protocol (HIT-RT) without any other type of exercise, be it in parallel or during warm-up or cool down. Our HIT-RT protocol focused on the application of frequent phases of high intensity (... up to $85 \% 1 \mathrm{RM}$ ), high effort ${ }^{28,29}$ and/or high movement-velocity without any training breaks for 18 months. Two consistently supervised exercise sessions per week with 12-14 exercises/session from a pool of 18 exercises (calf raises, leg press, -extension, -curls, adduction, -abduction, hip extension, latissimus front pulleys, pull-overs, seated rowing, back extension, inverse fly, bench press, military press, lateral raises, butterfly with extended arms, crunches, lateral crunches) exclusively on machines (MedX, Ocala, USA) were conducted in a well- equipped gym (Kieser Training, Erlangen, Germany). After 12 weeks of familiarization and conditioning, the exercise training was structured into 8-12 week phases that included two or three linearly periodized 4-week mesocycles with each 4th week as a regeneration week. Relative intensity during the mesocycles varied between $60 \%$ and $85 \%$ 1RM; after 4 months of exercise, in about $40-65 \%$ of the sets/session the number of repetition per set was conducted to repetition maximum. ${ }^{29}$ Supersets and drop-sets were used to further intensify the exercise protocol, however, after 13-14 months of exercise no further changes of the exercise protocol were intended.

\section{Study Outcomes}

During the FrOST 6-month detraining-FU, all but one previous outcomes of FrOST were evaluated. However, due to logistic problems and radiation protection specifications, we were unable to evaluate integrated BMD of the LS as assessed by quantitative computed tomography (QCT). In this contribution, we focus on body composition and cardiometabolic health.

\section{Study Outcomes}

Main outcome of the present study

- Lean body mass as determined by Dual-Energy x-ray Absorptiometry (DXA)

Secondary study outcomes

- Total body fat rate (\%) as determined by DXA

- Abdominal body fat rate (\%) as determined by DXA

- Metabolic Syndrome Z-Score based on the definition of the International Diabetes Federation (IDF). ${ }^{30}$

No changes of trial outcomes were made after trial commencement.

\section{Assessments}

Assessments were conducted at baseline, after 6, 12, 18 months of intervention and after 6 months of detraining. We placed great emphasis on standardized assessments. This included all assessments and analyses being completed (a) by the same research assistant, (b) at the same time of the day $( \pm 2 \mathrm{~h})$, (c) at the identical location, (d) in the same order, (e) with the same calibrated devices and (f) with the same configuration of the device. Further, study 
participants were strictly asked to maintain their habitual diet and avoid intense physical activity during the two days prior to the assessments.

Body height was determined by a Holtain stadiometer (Crymych Dyfed, Great Britain). Body mass was evaluated by the scale function of a direct-segmental, multifrequency Bio-Impedance-Analysis (DSM-BIA; InBody 770 , Seoul, Korea). Lean body mass, total and abdominal body fat percentage were determined with a DXA-scanner (QDR 4500a, Discovery-upgrade, Hologic Inc., Bedford, USA) according to the manufacturer's specifications. The "compare mode" was used to exactly reproduce baseline specifications during FU-assessments.

Representative for cardiometabolic risk, we calculated the metabolic syndrome Z-Score (MetSZ) based on the IDF-definition: ${ }^{30}$ [(40 - HDL-cholesterol)/SD HDL-C $]+$ [(triglycerides - 150)/SD TriGly $]+[($ Glucose -100$) / \mathrm{SD}$ Glucose $]+[($ waist circumference -88$) / \mathrm{SD}$ WC $]+[($ Mean arterial blood pressure - 100)/SD MAP]. For details on assessments of MetS-components the reader is kindly referred to another publication. ${ }^{31}$

Standardized questionnaires asked for several baseline and follow-up parameters. Baseline questionnaires solicit (a) demographic parameters, (b) diseases, limitations, injuries, operations, (c) pharmacologic agents, dietary supplements, (d) lifestyle, with specific emphasis on physical activity and exercise habits, ${ }^{32,33}$ (e) falls and low trauma fractures during the previous year, and (f) independence status. The follow-up questionnaires focus on potential changes with impact on our study result. This includes in particular injuries, operations, changes of medication, complaints or diseases and physical activity. Very high emphasis was placed on the detection of changes of exercise and physical activity habits during the detraining period. In order to properly evaluate intensity and volume of physical activity and in particular exercise we used our physical activity and exercise questionnaire $^{32,33}$ specifically developed to determine mechanical loading. In order to generate high consistency, completeness and accuracy, the primary investigator lastly checked the FU-questionnaire in close interaction with the participants.

In order to monitor dietary intake and to calculate individual whey protein doses, all participants completed dietary records (Freiburger Nutrition Record, nutriscience, Hausach, Germany) at baseline and FU 6, 12, 18 and 24 months. Participants were asked to record 3 weekdays and one weekend day representative for their dietary habits. Protocols were analyzed consistently by the same research assistant.

\section{Statistical Analysis}

We conducted an Intention-to-treat (ITT) analysis that included all participants who were randomly assigned to the study arms at baseline. Missing data was imputed using $\mathrm{R}$ statistics software ( $\mathrm{R}$ Development Core Team Vienna, Austria) in combination with Amelia II. ${ }^{34}$ Imputation was repeated 100 times using the full data set for the multiple imputation procedure. Imputation diagnostic plots indicate that imputation worked well. To compare the changes of the detraining period between the $\mathrm{CG}$ and the HIT-RT (ie time-group interactions = effects) we applied an ANCOVA that adjusted for 18-month data of the corresponding parameter using group as covariate. The changes from baseline and to the end of the detraining period were also analyzed by an ANCOVA that adjusted for baseline data of the corresponding parameter and again included group as covariate. The within-group changes over time were analyzed by paired $t$-tests. Within- and between-imputation variances were analyzed using the approach suggested by Rubin ${ }^{35}$ and Barnard and Rubin. ${ }^{36}$ We consistently applied 2-tailed tests and accepted significance at $\mathrm{p}<0.05$.

\section{Results}

\section{Participant and Exercise Characteristics}

Participants' baseline and 18-month FU characteristics (ie baseline values of the detraining period) are displayed in Table 1. Applying a DXA-based cutoff point of $27 \%$ body fat rate to diagnose obesity ${ }^{37}$ the vast majority of our cohort $(93 \%)$ can be considered as obese.

Baseline but not 18-month dietary protein intake varied significantly between the groups. Further, we observed a significant increase $(\mathrm{p}<0.001)$ in $25-\mathrm{OHD}$ concentration in both groups during the interventional period, indicating that cholecalciferol supplementation was successful in most subjects.

Three participants quit the study during the intervention phase and were also lost for 6-month detraining-FU (Figure 1). The two participants of the $\mathrm{CG}$ who were unable to attend the 18-month FU assessment were evaluated at 6-month detraining-FU. Two HIT-RT participants and one participant of the $\mathrm{CG}$ refused to visit the detraining-FU, due to fear of being infected during the assessments. 
Table I Characteristics of the Participants of the High-Intensity Resistance Exercise Training (HIT-RT) and Control Group (CG) at Study Start and After 18 Months of Intervention

\begin{tabular}{|c|c|c|c|c|}
\hline \multirow[t]{3}{*}{ Variable } & \multicolumn{2}{|c|}{ CG $(n=22)$} & \multicolumn{2}{|c|}{ HIT-RT (n=2I) } \\
\hline & Baseline & I 8 Month & Baseline & I8 Month \\
\hline & MV \pm SD & MV \pm SD & $M V \pm S D$ & MV \pm SD \\
\hline Age [years] & $79.2 \pm 4.7$ & $80.8 \pm 4.7$ & $77.8 \pm 3.6$ & $79.6 \pm 3.6$ \\
\hline Body Mass Index $\left[\mathrm{kg} / \mathrm{m}^{2}\right]$ & $24.5 \pm 1.9$ & $24.6 \pm 2.1$ & $25.0 \pm 3.0$ & $24.8 \pm 3.0$ \\
\hline Total Body Fat [\%] & $34.2 \pm 6.1$ & $32.2 \pm 5.5$ & $33.6 \pm 4.0$ & $33.5 \pm 4.3$ \\
\hline More than two diseases $[\mathrm{n}]^{\mathrm{a}}$ & 12 & 13 & 10 & 9 \\
\hline Metabolic Syndrome $[\mathrm{n}]^{\mathrm{b}}$ & 12 & 12 & 11 & 9 \\
\hline Diabetes Mellitus type II [n] & 1 & I & 1 & 1 \\
\hline Physical activity [Index $]^{c}$ & $4.15 \pm 1.53$ & $4.32 \pm 1.44$ & $4.45 \pm 1.32$ & $4.51 \pm 1.27$ \\
\hline Exercise volume [min/week] & $59 \pm 56$ & $54 \pm 56$ & $42 \pm 52$ & $52 \pm 50$ \\
\hline $25-\mathrm{OHD}$ level $[\mathrm{ng} / \mathrm{mL}]^{\mathrm{de}}$ & $17.5 \pm 7.0$ & $29.6 \pm 5.8$ & $21.6 \pm 8.4$ & $28.1 \pm 5.8$ \\
\hline Calcium intake $(\mathrm{mg} / \mathrm{d})^{\mathrm{e}}$ & $833 \pm 282$ & $852 \pm 266$ & $802 \pm 226$ & $827 \pm 291$ \\
\hline Energy intake $[\mathrm{kcal} / \mathrm{d}]^{f}$ & $2291 \pm 590$ & $2242 \pm 639$ & $2155 \pm 416$ & $2197 \pm 508$ \\
\hline Dietary Protein intake $[\mathrm{g} / \mathrm{kg} / \mathrm{d}]^{f}$ & $1.29 \pm 0.24$ & $1.25 \pm 0.23$ & $1.10 \pm 0.25$ & $1.15 \pm 0.27$ \\
\hline
\end{tabular}

Notes: ${ }^{a}$ ICD-10 based disease cluster of Schäfer et al ${ }^{38}$ bas defined by the International Diabetes Federation; ${ }^{30}$ "Scale from (I) "very low" to (7) "very high"32 dRoche Diagnostics, Mannheim, Germany; ${ }^{\mathrm{A} A s}$ determined by a Calcium Questionnaire provided by Rheumaliga, Switzerland; ${ }^{\mathrm{f}} \mathrm{As}$ determined by dietary records.

Abbreviations: MV, mean value; SD, standard deviation; 25-OHD, calcidiol.

\section{Study Outcomes}

Figure 2 shows intervention and detraining effects on LBM in the HIT-RT and CG. After 18 months of intervention LBM increased significantly in the HIT-RT $(p<0.001)$ and decreased slightly in the $\mathrm{CG}(\mathrm{p}=0.11)$. Intervention effect (ie group differences in 18-month changes) was significant $(\mathrm{p}<0.001 ; 1.73 \mathrm{~kg}, 95 \%$-CI: $1.13-2.32 \mathrm{~kg})$. During the detraining period, LBM gains of the HIT RT were nearly halved $(\mathrm{p}<0.001)$, but still remained significantly $(p<0.001)$ above baseline (Figure 2$)$. In parallel, LBM of the CG decreased further during the detraining period, resulting in a significant 24-month overall change from baseline $(\mathrm{p}=0.007)$. Briefly, LBM mass reduction during the detraining period was significantly more pronounced in the HIT-RT compared with the CG $(\mathrm{p}<0.001)$. However, even after 6 months of detraining the intervention effect remained significant $(1.04 \mathrm{~kg}, 0.46-1.59 \mathrm{~kg}$, $\mathrm{p}<0.001)$. In summary, we thus confirmed our hypothesis (1.1) that 6 months of detraining after 18 months of HITRT significantly decrease LBM compared to the nontraining control group (Figure 2). However, even after this significant 6-month detraining effect, we observed a significant training effect at study end, thus we reject our hypothesis (2.1).

Figures 3 and 4 display changes of total and abdominal body fat rate (\%) during training and detraining in the HIT-RT and CG. Both fat proportions increased in the CG during the 24-month study period (total body fat:

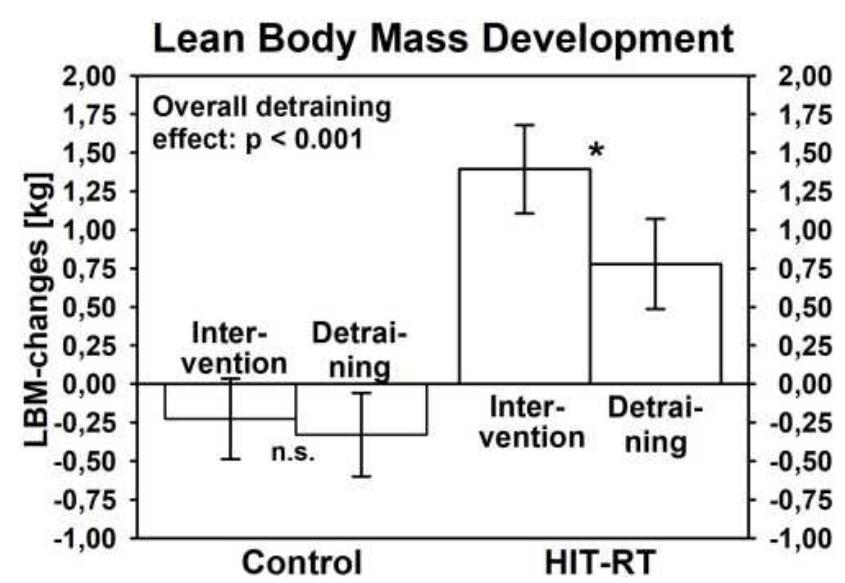

Figure 2 Mean values and $95 \%-\mathrm{Cl}$ for changes of lean body mass after training and detraining in the HIT-RT and CG.

Note: ${ }^{*} \mathrm{p}<0.001$.

Abbreviation: n.s., non-significant. 


\section{Total Body Fat Rate Development}

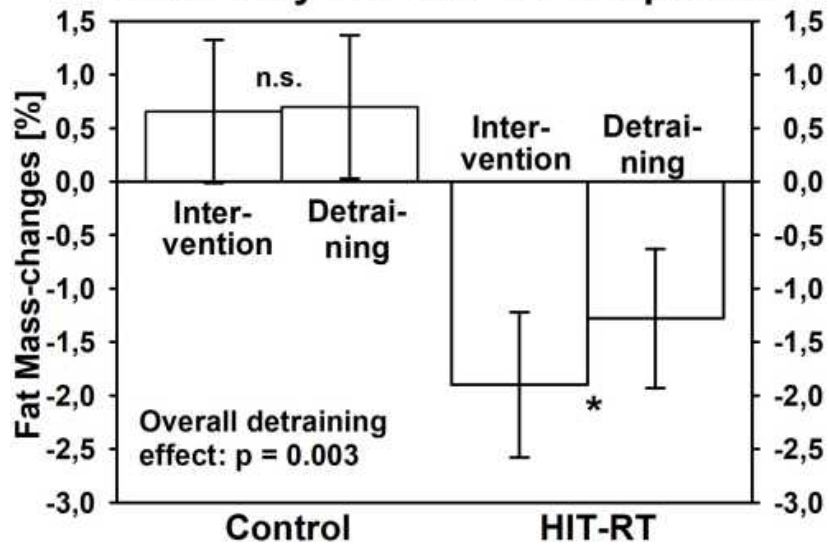

Figure 3 Mean values and $95 \%-\mathrm{Cl}$ for changes of total body fat rate after training and detraining in the HIT-RT and CG.

Note: ${ }^{*} \mathrm{p}=0.015$.

Abbreviation: n.s., non-significant.

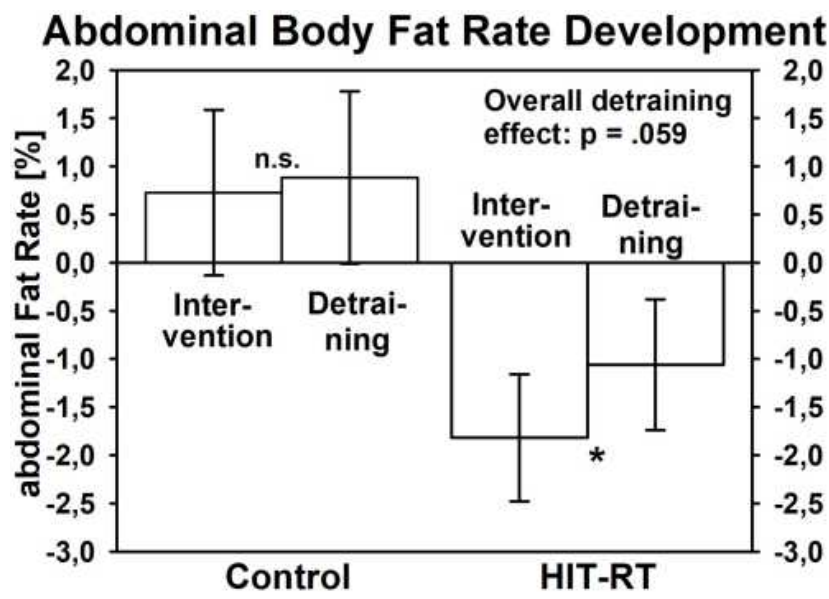

Figure 4 Mean values and $95 \%-\mathrm{Cl}$ for changes of abdominal body fat rate after training and detraining in the HIT-RT and CG.

Note: ${ }^{*} \mathrm{p}=0.007$

Abbreviation: n.s., non-significant.

$\mathrm{p}=0.048$; abdominal body fat: $\mathrm{p}=0.053$ ). In parallel, in the HIT-RT group total and abdominal fat decreased significantly (both $\mathrm{p}<0.001$ ) during the intervention period and increased significantly for total $(\mathrm{p}=0.015)$ and abdominal $(p=0.007)$ body fat rate during the detraining period. Detraining effects differ significantly between the groups for total $(p=0.003)$ but not for abdominal $(p=0.059)$ body fat. Effects that were significant after the end of the intervention (total body fat: $2.54 \%, 95 \%$-CI: $1.90-3.21 \%$; abdominal body fat: $2.66 \%, 1.70-3.60 \%$; both $\mathrm{p}<0.001$ ) remained significant after 6 months of detraining (total body fat: $1.99 \%, 1.25-2.77 \%$; abdominal body fat: $1.94 \%, 0.91-2.99 \%$, both $\mathrm{p} \leq .001$ ) (Figures 2 and 3 ). However, in summary we accepted hypothesis (1.2) and rejected hypothesis (1.3) of a significantly more pronounced detraining effect in the HIT-RT vs the control group on total body fat and abdominal body fat rate. We further reject our secondary hypotheses ((2.2) and (2.3)) that the significant HIT-RT effects on total and abdominal body fat are lost after 6 months of detraining.

Changes of the MetSZ during training and detraining are displayed in Table 2. MetSZ of the CG deteriorated non-significantly $(\mathrm{p}=0.080)$ during the intervention and detraining period (Table 1). In contrast, MetSZ in the HITRT improved significantly $(\mathrm{p}<0.001)$ during the interventional and decreased significantly $(p=0.002)$ during the detraining period. Detraining effects differ significantly between the groups $(p=0.003)$. The significant effect observed after 18 months of exercise $(\mathrm{p}=0.001)$ was lost after 6 months of detraining ( $\mathrm{p}=0.441)$. In summary, however, we confirmed hypothesis (1.4) of a significantly more pronounced detraining effect in the HIT-RT compared with the CG. Additionally, we confirmed our hypothesis (2.4).

In detail, all components of the MetSZ improved during the intervention, but only results on waist circumference, MAP and HDL-C showed significant training effects. ${ }^{31}$ Detraining changes of waist circumference, MAP and Triglycerides (all $\mathrm{p}>0.188$ ) in the HIT-RT did not differ significantly from the CG. However, we observed a significant detraining effect for HDL-C $(p=0.017)$ with significant HDL-C declines in the HITRT ( $\mathrm{p}=0.029)$. In contrast, glucose levels remained stable in the HIT-RT, but declined considerably in the CG resulting in a significant detraining effect $(p=0.040)$. At study end, only the effect for waist circumference remained statistically significant $(\mathrm{p}<0.001)$.

\section{Confounding Parameters}

According to our physical activity questionnaire, ${ }^{33}$ habitual physical activity did not vary significantly in the EG and $\mathrm{CG}$ over the intervention period (Table 1). Habitual exercise volume was also stable during the intervention period. However, both groups increased the amount of individual aerobic outdoor activities, ie walking and/or biking (20 $\pm 29 \%, \mathrm{p} \geq .103)$ during the detraining period. This can be largely attributed to the COVID-19 induced restrictions, with individual outdoor activities still being allowed. None of the participants attended supervised group exercise training or resistance-type exercise training during the six-month observational (detraining) period of the present study. 
Table 2 Mean Values and 95\%-Cl for Changes of the Metabolic Syndrome Z-Score After Training and Detraining in the CG and HITRT Group

\begin{tabular}{|c|c|c|c|c|}
\hline & CG & HIT-RT & Difference & \multirow[t]{2}{*}{ p-value } \\
\hline & MV (95\%-Cl) & MV (95\%-Cl) & MV (95\%-Cl) & \\
\hline \multicolumn{5}{|l|}{ Metabolic Syndrome Z-Score [Z] } \\
\hline Baseline & $-3.99(-2.65$ to -5.33$)$ & $-2.89(-1.70$ to -4.07$)$ & $\longrightarrow$ & 0.355 \\
\hline 18-month changes ${ }^{\mathrm{a}, \mathrm{b}, \mathrm{c}}$ (intervention) & $0.63(-0.07$ to 1.33$)$ & $-0.87(-0.20$ to -1.53$)$ & $1.50(0.60$ to $2.5 \mathrm{I})$ & 0.001 \\
\hline 24-month changes ${ }^{\mathrm{a}, \mathrm{b}, \mathrm{d}}$ (detraining) & $0.62(-0.1 \mid$ to 1.36$)$ & $0.10(-0.57$ to 0.77$)$ & $0.52(-0.93$ to 2.10$)$ & $0.44 \mid$ \\
\hline
\end{tabular}

Notes: ${ }^{a}$ Changes from baseline, ${ }^{b}$ of importance for the interpretation of the results, negative Z-Score changes can be considered as favorable, ${ }^{c}$ adjusted on baseline differences, dadjusted on 18-months differences.

Dietary calcium and protein intake did not vary significantly over the two-year period. However, due to the different recommendations ${ }^{26}$ on total protein intake in $\mathrm{CG}$ and HIT-RT (1.2 vs $1.5-1.6 \mathrm{~g} / \mathrm{kg} / \mathrm{d})$, total protein intake of the HIT-RT decreased after cessation of WPS by $0.35--$ $0.45 \mathrm{~g} / \mathrm{kg} / \mathrm{d}$, while on average no relevant changes in total protein intake were recorded for the CG. As reported, cholecalciferol supplementation was maintained during the detraining period, and hence 25-OHD levels did not change significantly during the detraining period (HIT-RT: $28.1 \pm 6.1$ to $30.3 \pm 7.5 \mathrm{ng} / \mathrm{mL}$; CG: $29.6 \pm 5.8$ to $30.8 \pm 6.6 \mathrm{ng} /$ $\mathrm{mL}$ ). None of the participants reported injuries, operations, diseases, periods of physical inactivity $>1$ week, or changes of pharmacologic therapy with impact on muscle or bone metabolism during the detraining period.

\section{Discussion}

The present 18-month RT exercise study clearly substantiates the deleterious effects of 6 months of detraining on LBM, total and abdominal body fat rate and the MetSZ-Score in our predominately obese cohort of cdw men 72-91 years old with low muscle and bone mass. However, although 6 months of detraining reduced the training effect of an 18-month HIT-RT protocol, apart from the METS-Z-Score, the effects remained significant after the detraining period (ie after 24 months). We speculate that this sustainability can be attributed to the long intervention period, ie to the phenomenon that adaptation achieved over a longer period might be characterized by a lower susceptibility to failure ("adaptation stability"39). Of importance for the present detraining issue, the cessation of exercise refers exclusively to the dedicated HIT-RT, while the volume of outdoor physical activity and exercise training (ie brisk walking, Nordic walking, cycling) increased in both groups during the detraining period.
One may argue that in addition to the cessation of (RT) exercise, the termination of whey protein supplementation (WPS) might have contributed to the significant deterioration not only of body composition but also in cardiometabolic risk factors. Indeed, some studies suggested evidence for a positive effect of isolated protein and amino acid supplementation on LBM (e.g. ${ }^{40-45}$ ), body fat (e.g. ${ }^{46,47}$ ) and cardio-metabolic risk factors (review $\mathrm{in}^{48}$ ) in older adults. We are unable to separate the exercise vs WPS effects on our outcomes during training ${ }^{18,31}$ and detraining; however, (1) based on the rather high dietary protein intake (Tables 1 and 2) considering that the $1.5 \mathrm{~g} / \mathrm{d} / \mathrm{kg}$ WPS of the HIT-RT was derived from recommendations of the PROT-AGE group ${ }^{26}$ for exercising older adults, we speculate that the cessation of WPS after the end of the intervention had only a minor additional impact on our result of detraining effects on body composition and cardiometabolic risk.

A meaningful comparison of our result on detraining effects on body composition and cardiometabolic health in older people with other studies is far from trivial. Considering that apart from age ${ }^{3,49}$ particularly the (1) length of the intervention period (e.g. $\left.{ }^{39}\right)$, (2) type, intensity and volume of the exercise applied (e.g..$^{3,7,15,50,51}$ ), (3) length of the detraining period (e.g. ${ }^{3,52}$ ) and (4) activity during the detraining period contribute considerably to the detraining result of a given outcome ( $\ldots$ that might be also more or less sensitive to detraining, ${ }^{8,50}$ ), inconsistency of detraining study results become comprehensible. Applying 5 cycles of 9 months of RT intermitted by 3 months of detraining each, although predominately non-significant, Douda et $\mathrm{al}^{4}$ consistently reported reversions of training effects on LBM and body fat during the detraining periods in their cohort of women 60 years and older. After 24 weeks of RT-training and detraining each, Hakkinen et $\mathrm{al}^{8}$ reported a significant 
detraining effect on cross-sectional area of the quadriceps femoris in younger and older men, that average below baseline. Applying a RT and detraining protocol for 24 weeks each in people $65-83$ years old, Taaffe et $\mathrm{al}^{14}$ found significant detraining effects on LBM and body fat. The authors additionally reported a significant increase of fatty infiltration of thigh muscles during detraining. Applying an albeit shorter RT period of 16 weeks, Bickel et al. ${ }^{3}$ reported significant reductions in thigh lean mass that dropped below baseline values already after 8 weeks of detraining. On the other hand, the authors observe a complete reversal of the training-induced myofiber type IIx to IIa shift not before 32 weeks of detraining.

Detraining effects on cardiometabolic risk factors after RT are very rarely evaluated in older people.$^{53,54}$ Further, no RT study with older people focused on detraining effects on the MetS-Z-Score. Additionally, most studies on RT (or at least with an RT-component) applied short intervention ( $\leq 16$ weeks) and/or short detraining ( $<12$ weeks) periods (e.g. ${ }^{5,53,55-59}$ ). However, a summary of (very) roughly comparable detraining studies provided inconsistent results on cardiometabolic risk factors. Applying 8 months of RT in coronary artery disease (CAD) patients $62 \pm 8$ years old, Theodorou et $\mathrm{al}^{52}$ reported no significant detraining effects for HDL-C or Triglycerides after 1,2 or 3 months of detraining. Leitao et $\mathrm{al}^{54}$ who prescribed multicomponent exercise with an RT component for 9 months listed significant detraining effects on systolic and diastolic blood pressure and Triglycerides after 3 months of detraining in women $60-75$ years old. Takmakidis and Volaklis ${ }^{60}$ confirmed these unfavorable results on Triglycerides after 8 months of training and 3 months of detraining in men $57 \pm 10$ years old with CAD.

Summing up the features and limitations of the present study on detraining, the main limitation might be the nonpreplanned detraining approach. After the 18-month study intervention, we did not intend to tell our aged participants that they had to stop exercising (.... which would be a morally reprehensible approach), but aimed to continue the RT-exercise training in a sustainable setting. However, our inability to quickly reorganize the training and the subsequent COVID-19 related lockdown offered the opportunity for the present detraining study. Due to the initially nonintended detraining period, we supplemented all participants with a further 180 capsules each of 2500 IE cholecalciferol from our remaining stocks after the end of the intervention. Additionally, related to the lack of an a priori sample size analysis for the detraining approach, there is some evidence that our approach is underpowered to successfully address some of the study outcomes. Another biometrical issue was that following the recommendations of $\mathrm{Li}^{61}$ we do not adjust on secondary outcomes, thus the reader should be aware of potential multiplicity. This feature might be further aggravated since we addressed different endpoints of the detraining aspect. As the decisive outcome, we consider differences in detraining changes between HIT-RT and CG. However, we also listed changes during detraining in the HIT-RT, as well as baseline-adjusted overall effects after 24 months. In contrast, most other studies simply focus on detraining changes in the exercise group without considering adjuvant changes in the CG. Lastly, we selected LBM and not, as initially registered, skeletal muscle mass index as the primary study outcome, in order to increase the comparability with other trials.

\section{Conclusion}

Addressing the generalization of our results on detraining is a daunting task. Although our cohort of predominately obese, 72-91-year-old HIT-RT completers with osteosarcopenia might not be "fully representative" for older cdw cohorts, we nevertheless think the aspects listed below can be generalized. Hence, addressing the clinical relevance of our results, we conclude that (1) although training breaks must be long enough to allow full regeneration of the system(s) addressed, 6 months of detraining result in significant and clinically relevant negative effects on body composition and cardiometabolic outcomes and should therefore be avoided. (2) Detraining effects after abrupt cessation of (HIT-)RT cannot be compensated by maintaining or slightly increasing physical activity with an aerobic character (ie walking, cycling). (3) Nevertheless, there is some evidence that adaptation achieved over a longer period of time (ie 18 months) is characterized by higher stability and lower susceptibility to failure ${ }^{39}$ during detraining. Correspondingly, exercise training protocols particularly for older people should focus on continuous exercise protocols with short training breaks.

\section{Data Sharing Statement}

The data that support the findings of this study are available from the corresponding author (WK), upon reasonable request.

\section{Author Contributions}

WK, DS, MK, SvS made a significant contribution to the work reported, whether that is in the conception, study design, execution, acquisition of data, analysis and interpretation, or 
in all these areas. WK, DS, MK, SvS have drafted or written, or substantially revised or critically reviewed the article and have agreed on the journal to which the article will be submitted. Further, all authors reviewed and agreed on all versions of the article before submission, during revision, the final version accepted for publication, and any significant changes introduced at the proofing stage. Finally, WK, DS, MK, SvS agree to take responsibility and be accountable for the contents of the article.

\section{Funding}

The authors are very grateful to the support of the Universitäts-Bund of the University of ErlangenNürnberg, Erlangen that provided resources for the 6-month follow-up assessment. The authors further acknowledge the support of Kieser Training (Erlangen, Germany) which provided the gym facilities and exercise supervision support. The authors also acknowledge the contribution of the Institute of Radiology, FAU, Erlangen, Germany.

\section{Disclosure}

The authors report no conflicts of interest in this work.

\section{References}

1. Eichberg S, Rott C. Sportverhalten im mittleren und höheren Erwachsenenalter. Bedingungsfaktoren für Kontinuität und Diskontinuität. J Public Health. 2004;12:93-104. doi:10.1007/ s10389-004-0020-y

2. Pahmeier I. Sportliche Aktivität aus der Lebensperspektive. Z Gerontol Geriat. 2008;41:168-176. doi:10.1007/s00391-008-0543-x

3. Bickel CS, Cross JM, Bamman MM. Exercise dosing to retain resistance training adaptations in young and older adults. Med Sci Sports Exerc. 2011;43(7):1177-1187. doi:10.1249/MSS.0b013e318207c15d

4. Douda HT, Kosmidou KV, Smilios I, Volaklis KA, Tokmakidis SP. Community-based training-detraining intervention in older women: a five-year follow-up study. J Aging Phys Act. 2015;23(4):496-512. doi:10.1123/japa.2013-0241

5. Elliott KJ, Sale C, Cable NT. Effects of resistance training and detraining on muscle strength and blood lipid profiles in postmenopausal women. $B r \quad J$ Sports Med. 2002;36(5):340-344. doi:10.1136/ bjsm.36.5.340

6. Esain I, Gil SM, Bidaurrazaga-Letona I, Rodriguez-Larrad A. Effects of 3 months of detraining on functional fitness and quality of life in older adults who regularly exercise. Aging Clin Exp Res. 2019;31 (4):503-510. doi:10.1007/s40520-018-0990-1

7. Fatouros IG, Kambas A, Katrabasas I, et al. Strength training and detraining effects on muscular strength, anaerobic power, and mobility of inactive older men are intensity dependent. Br J Sports Med. 2005;39(10):776-780. doi:10.1136/bjsm.2005.019117

8. Hakkinen K, Alen M, Kallinen M, Newton RU, Kraemer WJ. Neuromuscular adaptation during prolonged strength training, detraining and re-strength-training in middle-aged and elderly people. Eur J Appl Physiol. 2000;83(1):51-62. doi:10.1007/ s004210000248
9. Henwood TR, Taaffe DR. Detraining and retraining in older adults following long-term muscle power or muscle strength specific training. J Gerontol a Biol Sci Med Sci. 2008;63(7):751-758. doi:10.1093/gerona/63.7.751

10. Lovell DI, Cuneo R, Gass GC. The effect of strength training and short-term detraining on maximum force and the rate of force development of older men. Eur J Appl Physiol. 2010;109(3):429-435. doi:10.1007/s00421-010-1375-0

11. Porter MM, Nelson ME, Singh MAF, et al. Effects of long-term resistance training and detraining on strength and physical activity in older women. JAPA. 2002;10(3):260-270. doi:10.1123/ japa.10.3.260

12. Sakugawa RL, Moura BM, Orssatto L, Bezerra ES, Cadore EL, Diefenthaeler F. Effects of resistance training, detraining, and retraining on strength and functional capacity in elderly. Aging Clin Exp Res. 2019;31(1):31-39. doi:10.1007/s40520-018-0970-5

13. Snijders T, Leenders M, de Groot L, van Loon LJC, Verdijk LB. Muscle mass and strength gains following 6months of resistance type exercise training are only partly preserved within one year with autonomous exercise continuation in older adults. Exp Gerontol. 2019;121:71-78. doi:10.1016/j.exger.2019.04.002

14. Taaffe DR, Henwood TR, Nalls MA, Walker DG, Lang TF, Harris TB. Alterations in muscle attenuation following detraining and retraining in resistance-trained older adults. Gerontology. 2009;55(2):217-223. doi:10.1159/000182084

15. Tokmakidis SP, Kalapotharakos VI, Smilios I, Parlavantzas A. Effects of detraining on muscle strength and mass after high or moderate intensity of resistance training in older adults. Clin Physiol Funct Imaging. 2009;29(4):316-319. doi:10.1111/j.1475097X.2009.00866.x

16. Zech A, Drey M, Freiberger E, et al. Residual effects of muscle strength and muscle power training and detraining on physical function in community-dwelling prefrail older adults: a randomized controlled trial. BMC Geriatr. 2012;12:68. doi:10.1186/1471-2318-12-68

17. Kemmler W, Kohl M, Froehlich M, Engelke K, von Stengel S, Schoene D. Effects of high intensity resistance training on fitness and fatness in older men with osteosarcopenia. Front Physiol. 2020;11:1014. doi:10.3389/fphys.2020.01014

18. Kemmler W, Kohl M, Jakob F, Engelke K, von Stengel S. Effects of high intensity dynamic resistance exercise and whey protein supplements on osteosarcopenia in older men with low bone and muscle mass. Final results of the randomized controlled FrOST study. Nutrients. 2020;12(8):2341. doi:10.3390/nu12082341

19. Kemmler W, Kohl M, Frohlich M, Schoene D, von Stengel S. Detraining effects after 18 months of high intensity resistance training on osteosarcopenia in older men-Six-month follow-up of the randomized controlled Franconian Osteopenia and Sarcopenia Trial (FrOST). Bone. 2021;142:115772. doi:10.1016/j.bone.2020.115772

20. World Medical Association. World Medical Association declaration of Helsinki: ethical principles for medical research involving human subjects. JAMA. 2013;310(20):2191-2194. doi:10.1001/ jama.2013.281053

21. Lichtenberg T, von Stengel S, Sieber C, Kemmler W. The favorable effects of a high-intensity resistance training on sarcopenia in older community-dwelling men with osteosarcopenia: the randomized controlled FrOST study. Clin Interv Aging. 2019;14:2173-2186. doi: $10.2147 /$ CIA.S225618

22. Kemmler W, Kohl M, Frohlich M, et al. Effects of high intensity resistance training on osteopenia and sarcopenia parameters in older men with osteosarcopenia - one-year results of the randomized controlled Franconian Osteopenia and Sarcopenia Trial (FrOST). J Bone Miner Res. 2020;35(9):1634-1644. doi:10.1002/jbmr.4027

23. Baumgartner RN, Koehler KM, Gallagher D, et al. Epidemiology of sarcopenia among the elderly in New Mexico. Am J Epidemiol. 1998;147(8):755-763. doi:10.1093/oxfordjournals.aje.a009520 
24. Cruz-Jentoft AJ, Baeyens JP, Bauer JM, et al. Sarcopenia: European consensus on definition and diagnosis: report of the European Working Group on sarcopenia in older people. Consensus development conference practice guideline research support, non-U.S. Gov't Age Ageing. 2010;39(4):412-423. doi:10.1093/ageing/afq034

25. World Health Organization. Assessment of osteoporotic fracture risk and its application to screening for postmenopausal osteoporosis. 1994: WHO technical report series No. 843. 1994.

26. Bauer J, Biolo G, Cederholm T, et al. Evidence-based recommendations for optimal dietary protein intake in older people: a position paper from the PROT-AGE Study Group. J Am Med Dir Assoc. 2013;14(8):542-559. doi:10.1016/j.jamda.2013.05.021

27. DVO. Prophylaxe, Diagnostik Und Therapie Der OSTEOPOROSE Bei Postmenopausalen Frauen Und Bei Männern Schattauer. Schattauer Verlag; 2017.

28. Zourdos MC, Klemp A, Dolan C, et al. Novel resistance training-specific rating of perceived exertion scale measuring repetitions in reserve. J Strength Cond Res. 2016;30(1):267-275. doi:10.1519/JSC.0000000000001049

29. Steele J, Fisher J, Giessing J, Gentil P. Clarity in reporting terminology and definitions of set end points in resistance training. Muscle Nerve. 2017;56(3):368-374. doi:10.1002/mus.25557

30. Alberti KG, Zimmet P, Shaw J. Metabolic syndrome-a new worldwide definition. A consensus statement from the International Diabetes Federation. Diabet Med. 2006;23(5):469-480. doi:10.1111/ j.1464-5491.2006.01858.x

31. Kemmler W, Kohl M, von Stengel S, Schoene D. Effect of high-intensity resistance exercise on cardiometabolic health in older men with osteosarcopenia. The randomized controlled Franconian Osteopenia and Sarcopenia Trial (FrOST). BMJ OPEN. 2020; accepted for publication.

32. Kemmler W, Weineck J, Kalender WA, Engelke K. The effect of habitual physical activity, non-athletic exercise, muscle strength, and VO2max on bone mineral density is rather low in early postmenopausal osteopenic women. J Musculoskelet Neuronal Interact. 2004;4(3):325-334.

33. Schoeffl I, Kemmler W, von Stengel S, Engelke K, Kalender W, Engelke K. In healthy elderly postmenopausal women variations in BMD and BMC at various skeletal sites are associated with differences in weight and lean body mass rather than by variations in habitual physical activity, strength or $\mathrm{VO}_{2} \max$. $J$ Musculoskelet Neuronal Interact. 2008;8(4):363-374.

34. Honaker J, King G, Blackwell M. Amelia II: a program for missing data. JSS. 2011;45(7):1-47.

35. Rubin DB. Multiple Imputation for Nonresponse in Surveys. John Wiley and Sons; 1987.

36. Barnard J, Rubin DB. Small-sample degrees of freedom with multiple imputation. Biometrika. 1999;86(4):948-955. doi:10.1093/biomet/86.4.948

37. Baumgartner RN. Body composition in healthy aging. Ann NY Acad Sci. 2000;904:437-448. doi:10.1111/j.1749-6632.2000.tb06498.x

38. Schafer I, von Leitner EC, Schon G, et al. Multimorbidity patterns in the elderly: a new approach of disease clustering identifies complex interrelations between chronic conditions. PLoS One. 2010;5(12): e15941. doi:10.1371/journal.pone.0015941

39. Weineck J. Optimales Training. Spitta-Verlag; 2019.

40. Bauer JM, Verlaan S, Bautmans I, et al. Effects of a vitamin D and leucine-enriched whey protein nutritional supplement on measures of sarcopenia in older adults, the PROVIDE study: a randomized, double-blind, placebo-controlled trial. $J$ Am Med Dir Assoc. 2015;16(9):740-747. doi:10.1016/j.jamda.2015.05.021

41. Komar B, Schwingshackl L, Hoffmann G. Effects of leucine-rich protein supplements on anthropometric parameter and muscle strength in the elderly: a systematic review and meta-analysis. $J$ Nutr Health Aging. 2015;19(4):437-446. doi:10.1007/s12603-0140559-4
42. Malafarina V, Uriz-Otano F, Iniesta R, Gil-Guerrero L. Effectiveness of nutritional supplementation on muscle mass in treatment of sarcopenia in old age: a systematic review. J Am Med Dir Assoc. 2013;14 (1):10-17. doi:10.1016/j.jamda.2012.08.001

43. Xu ZR, Tan ZJ, Zhang Q, Gui QF, Yang YM. Clinical effectiveness of protein and amino acid supplementation on building muscle mass in elderly people: a meta-analysis. PLoS One. 2014;9(9):e109141. doi:10.1371/journal.pone.0109141

44. Xu ZR, Tan ZJ, Zhang Q, Gui QF, Yang YM. The effectiveness of leucine on muscle protein synthesis, lean body mass and leg lean mass accretion in older people: a systematic review and meta-analysis. $\mathrm{Br}$ J Nutr. 2015;113(1):25-34. doi:10.1017/S0007114514002475

45. Zhu K, Kerr DA, Meng X, et al. Two-year whey protein supplementation did not enhance muscle mass and physical function in well-nourished healthy older postmenopausal women. J Nutr. 2015;145(11):2520-2526. doi:10.3945/jn.115.218297

46. Baer DJ, Stote KS, Paul DR, Harris GK, Rumpler WV, Clevidence BA. Whey protein but not soy protein supplementation alters body weight and composition in free-living overweight and obese adults. J Nutr. 2011;141(8):1489-1494. doi:10.3945/ jn.111.139840

47. Tahavorgar A, Vafa M, Shidfar F, Gohari M, Heydari I. Whey protein preloads are more beneficial than soy protein preloads in regulating appetite, calorie intake, anthropometry, and body composition of overweight and obese men. Nutr Res. 2014;34(10):856-861. doi:10.1016/j.nutres.2014.08.015

48. Fekete AA, Givens DI, Lovegrove JA. Can milk proteins be a useful tool in the management of cardiometabolic health? An updated review of human intervention trials. Proc Nutr Soc. 2016;75 (3):328-341. doi:10.1017/S0029665116000264

49. Toraman NF. Short term and long term detraining: is there any difference between young-old and old people? $\mathrm{Br}$ J Sports Med. 2005;39(8):561-564. doi:10.1136/bjsm.2004.015420

50. Ivey FM, Tracy BL, Lemmer JT, et al. Effects of strength training and detraining on muscle quality: age and gender comparisons. $J$ Gerontol a Biol Sci Med Sci. 2000;55(3):B152-B159. doi:10.1093/gerona/55.3.B152

51. Slentz CA, Houmard JA, Johnson JL, et al. Inactivity, exercise training and detraining, and plasma lipoproteins. STRRIDE: a randomized, controlled study of exercise intensity and amount. J Appl Physiol. 2007;103(2):432-442. doi:10.1152/japplphysiol.01314.2006

52. Theodorou AA, Panayiotou G, Volaklis KA, et al. Aerobic, resistance and combined training and detraining on body composition, muscle strength, lipid profile and inflammation in coronary artery disease patients. Res Sports Med. 2016;24(3):171-184. doi:10.1080/ 15438627.2016.1191488

53. da Cunha Nascimento D, Tibana RA, Benik FM, et al. Sustained effect of resistance training on blood pressure and hand grip strength following a detraining period in elderly hypertensive women: a pilot study. Clin Interv Aging. 2014;9:219. doi:10.2147/CIA.S56058

54. Leitao L, Pereira A, Mazini M, et al. Effects of three months of detraining on the health profile of older women after a multicomponent exercise program. Int $J$ Environ Res Public Health. 2019;16(20):3881. doi:10.3390/ijerph16203881

55. Lovell DI, Cuneo R, Gass GC. Strength training improves submaximum cardiovascular performance in older men. J Geriatr Phys Ther. 2009;32(3):117-124. doi:10.1519/00139143-200932030-00007

56. Moraes MR, Bacurau RF, Casarini DE, et al. Chronic conventional resistance exercise reduces blood pressure in stage 1 hypertensive men. J Strength Cond Res. 2012;26(4):1122-1129. doi:10.1519/ JSC.0b013e31822dfc5e

57. Nikseresht M, Hafezi Ahmadi MR, Hedayati M. Detraining-induced alterations in adipokines and cardiometabolic risk factors after nonlinear periodized resistance and aerobic interval training in obese men. Appl Physiol Nutr Metab. 2016;41(10):1018-1025. doi:10.1139/apnm-2015-0693 
58. Nolan PB, Keeling SM, Robitaille CA, Buchanan CA, Dalleck LC. The effect of detraining after a period of training on cardiometabolic health in previously sedentary individuals. Int J Environ Res Public Health. 2018;15(10):2303. doi:10.3390/ijerph15102303

59. Rossi FE, Diniz TA, Neves LM, et al. The beneficial effects of aerobic and concurrent training on metabolic profile and body composition after detraining: a 1-year follow-up in postmenopausal women. Eur J Clin Nutr. 2017;71(5):638-645. doi:10.1038/ ejcn.2016.263
60. Tokmakidis SP, Volaklis KA. Training and detraining effects of a combined-strength and aerobic exercise program on blood lipids in patients with coronary artery disease. J Cardiopulm Rehabil. 2003;23 (3):193-200. doi:10.1097/00008483-200305000-00006

61. Li G, Taljaard M, Van den Heuvel ER, et al. An introduction to multiplicity issues in clinical trials: the what, why, when and how. Int J Epidemiol. 2017;46(2):746-755. doi:10.1093/ije/dyw320

\section{Publish your work in this journal}

Clinical Interventions in Aging is an international, peer-reviewed journal focusing on evidence-based reports on the value or lack thereof of treatments intended to prevent or delay the onset of maladaptive correlates of aging in human beings. This journal is indexed on PubMed Central, MedLine, CAS, Scopus and the Elsevier
Bibliographic databases. The manuscript management system is completely online and includes a very quick and fair peer-review system, which is all easy to use. Visit http://www.dovepress.com/ testimonials.php to read real quotes from published authors. 